\title{
THYROID DYSFUNCTIONS OF PREMATURITY AND IMPACTS ON NEURODEVELOPMENTAL OUTCOME
}

\author{
M.L. Chung ${ }^{1}$, H.W. Yoo ${ }^{2}$, K.-S. Kim ${ }^{2}$, I.Y. Sung ${ }^{3}$, S.-Y. Pi ${ }^{2}$, E.A.-R. Kim ${ }^{2}$ \\ ${ }^{1}$ Pediatrics, Haeundae Paik Hospital, University of Inje College of Medicine, Pusan, ${ }^{2}$ Pediatrics, ${ }^{3}$ Pediatric \\ Rehabilitation Medicine, Asan Medical Center, University of Ulsan College of Medicine, Seoul, Republic of \\ Korea
}

Background and aims: Thyroid dysfunction is very common among preterm infants and is associated with neurodevelopmental impairments.To determine the incidence and natural course of various thyroid dysfunctions and their impacts on neurodevelopmental outcomes of premature infants.

Methods: Infants of gestational age $<34$ weeks or whose birth weight was $<1,500 \mathrm{~g}$ were enrolled during 24 month study period $(n=177)$. We measured TSH and FT4 levels at $\leq 7$ days old, 4 weeks of age and prior to discharge. Furthermore, we assessed how various thyroid dysfunctions affected neurodevelopmental outcomes at 18 months of corrected age.

Results: Thyroid dysfunction was noted in 88 subjects. Hypothyroxinemia was observed in $23(13.0 \%)$ subjects and was more commonly observed in infants of lower GA and their thyroid function was influenced by variable clinical factors. Free T4 level was all normalized in these patients without thyroxine medication and neurodevelopmental outcome was not affected. In contrast, hyperthyrotropinemia was not associated with other clinical factors. Among 58 (32.8\%) subjects who had hyperthyrotropinemia, only 31 infants showed normal TSH levels at follow up tests. The remaining infants $(\mathrm{n}=27,15.3 \%)$ had persistently high TSH levels, which significantly impacted poor neurodevelopmental outcomes.

Conclusions: Thyroid dysfunction is common among preterm infants. With the exception of persistent hyperthyrotropinemia, it generally dose not affect neurodevelopmental outcomes. However, the beneficial effects of thyroid hormone therapy in patients with persistent hyperthyrotropinemia merits further study. 